\title{
Ittrium influence on exchange interactions in Laves phases $\left(\mathrm{Tb}_{1-x} \mathrm{Y}_{x}\right)_{0.8} \mathrm{Sm}_{0.2} \mathrm{Fe}_{2}$
}

\author{
Umkhaeva Z.S. \\ Metals, alloys \\ and composite materials laboratory \\ Kh. Ibragimov CI RAS \\ Grozny, RF \\ zargan.umhaeva@yandex.ru
}

Tereshina I.S.

Faculty of Physics

M.V. Lomonosov Moscow State University

Moscow, RF

irina_tereshina@mail.ru

\author{
Ilyushin A.S. \\ Metals, alloys \\ and composite materials laboratory \\ Kh. Ibragimov CI RAS \\ Grozny, RF \\ sols146i@phys.msu.ru
}

\author{
Aleroeva T.A. \\ Department of Physical Electronics \\ FSBEE HE Chechen State University \\ Grozny, RF \\ tamila_hinata.ru@mail.ru
}

\author{
Pankratov N.Yu. \\ General Physics and Magnetically-ordered \\ medium Department \\ M.V. Lomonosov Moscow State University \\ Moscow, RF \\ pankratov@phys.msu.ru
}

\begin{abstract}
The article provides the results of studying magnetic and magnetostrictive properties of alloys in multi component systems $\left(\mathrm{Tb}_{1-\mathrm{x}} \mathrm{Y}_{\mathrm{x}}\right)_{0.8} \mathrm{Sm}_{0.2} \mathrm{Fe}_{2}$ with the substitution parameter $\mathrm{x}=0,0.2,0.4,0.6,0.8,1$. Magnetization and magnetostriction studies are given in a wide temperature range. Compensation points on magnetization curves have been determined both along concentration and temperature curves. There is a magnetostriction constant sign inversion in the area of values $0.8 \leq x \leq 0.9$.
\end{abstract}

Keywords-Laves phases, magnetization, Curie temperature, magnetostriction

\section{INTRODUCTION}

Intermetallic compounds of rare-earth metals are widely known in science and techniques thanks to their unique physical properties. The most numerous classes of intermetallic compounds are compounds of $\mathrm{f}-\mathrm{p}$ - and d-metals. These compounds exist only in crystalline form; their physicchemical properties differ greatly from the properties of their components. Besides, intermetallides of a certain composition differ in their chemical properties from their metal components as well as from intermetallides of the same ultimate composition but with a different components ratio [1].

Among binary intermetallides the most available are:
- Kurnakov compounds (superstructures, ordered solid solutions),

- Laves phases,

- Hume-Rothery phases (electron compounds),

- $\sigma$-phases and $\sigma-$ like phases.

The most important compounds among the denoted ones, due to the search of new magnetic materials, are rare-earth elements compounds with $3 \mathrm{~d}$ - transitional elements $(\mathrm{Fe}, \mathrm{Co}$, $\mathrm{Ni}, \mathrm{Mn}$ ), in particular, such compounds as $\mathrm{RM}_{2}$. Stoichiometry $\mathrm{RM}_{2}$ (where $\mathrm{R}$ is a rare-earth element, $\mathrm{M}$ is an iron group element) relates to so called Laves phases, which occurs in two structural types: Laves cubic phase S15 and Laves hexagonal phase S14 [2]. These phases are relative to each other and in certain conditions there appear structural phase transitions between them. These are distortive phase transitions.

Laves phases combine relatively simple crystalline structure with unique magnetic properties, such as "huge" magnetostriction, rather high Curie temperatures [3] and big magnetocaloric effect [4]. Laves phases formation is restricted with atomic radii ratio of the constituent elements and valence electron concentration corresponding to one atom [5]. As a rule, there is no homogeneity range in intermetallides $R M 2$. 
Deviation from stoichiometry usually results in double-phase state and in some cases in structure type changes.

Besides, rare-earth intermetallic compounds, isotype to $\mathrm{S}$ 15 Laves phases undergo structural phase transitions during transitions into magneto ordered state with crystalline phase distortions. In $[6,7]$ there was information about low symmetric modifications in intermetallides $\mathrm{TbCo}_{2}, \mathrm{TbFe}_{2}$ and $\mathrm{TmFe}_{2}$. Spontaneous magnetostriction, with "gigantic" values $\sim 10^{-3}$ was the cause for crystalline distortions S15.

Among Laves phases $\mathrm{RM}_{2}$ there are intermetallides which together with greater spontaneous magnetostriction demonstrate gigantic magnetostriction induced with outer magnetic field [6].

So, Laves phases allow thorough theoretical and experimental study of structural phase changes regularities during magnetic ordering, and "gigantic" value of spontaneous magnetostriction provides possibility of quantitative evaluations of atomic crystalline structure distortions. Quasibinary systems study allows studying "spin flip-flops" and their influence on distortive phase transitions. Here, in the area of magnetic order-disorder transition, or in Curie temperature range, there appears significant magneto-caloric effect.

These peculiarities of Laves phases are determined by their magnetic nature. Many magnetic properties of such compounds are determined by three types of the exchanging interactions: between 3d-transition ions ( $\mathrm{Fe}-\mathrm{Fe}$ ); between rare-earth ions (R-R) and between rare-earth and 3d-transition ions (R-Fe). Rigorous theory of magnetic ordering in transitional d-metals is still developing.

Thanks to their magnetic properties Laves phases are perspective material for a new technique. In order to develop current fields of electrical technology, magnetic recording, computer engineering, micro - and nano-electronics, as well as techniques and physics where magnetic sensors are used (automobile manufacturing, magnetic flaw detection, medicine, tool engineering and others), the issue of receiving new type of magnetic materials is acute. That is why studying magnetic and magnetostriction properties of rare-earth metals with iron-group metals are acute as new type magnetic materials with controllable physic-mechanical properties are necessary.

It is important both for contemporary and innovative material science. The most suitable for technical application are intermetallic compounds of rare-earth elements with $\mathrm{Fe}$, as their unique magnetic properties are fulfilled at room temperatures. It makes synthesize and study quasi binary, quasi ternary and more complex systems on the base of rareearth compounds with iron with Laves phase structures.

\section{Methods AND MATERIALS}

Nowadays multi-component Laves phases are actual, combining elements composition in one of magnetic sub lattice; we can vary atomic spacing, and inter atomic exchange interaction resulting in this or that type of magnetic ordering. Creating new compositions with optimal physic-mechanical properties requires a detailed analysis of many alloys characteristics, as well as magnetic sub lattice occupancy influence on sign and degree of exchange interaction. These interactions determine magnetic, magnetostrictive and nuclearmagnetic properties of such compounds.

For this purpose we have synthesized alloys of new quasi ternary system $\left(\mathrm{Tb}_{1-\mathrm{x}} \mathrm{Y}_{\mathrm{x}}\right)_{0.8} \mathrm{Sm}_{0.2} \mathrm{Fe}_{2}$ with substitution parameter $\mathrm{x}=0,0.2,0.4,0.6,0.8,1.0$. The compounds $\mathrm{TbFe}_{2}$ and $\mathrm{SmFe}_{2}$ are the core of the system with record values of magnetostriction constants. There will be a conflict of exchange interaction in this system $\mathrm{Tb}-\mathrm{Fe}$ and $\mathrm{Sm}-\mathrm{Fe}$ depending on yttrium concentration, introduced into rare-earth sub lattice. That is why studying alloys of this system allows studying both exchange interaction between sub lattices R-Fe with dilution of rare-earth sub lattice with non-magnetic yttrium atoms, but also exchange interaction in rare-earth sub lattice itself. And exchange interactions, their type, sign and value determine all the range of unique magnetic, magnetostriction, and nuclear-magnetic properties of such compounds.

Alloys synthesis $\left(\mathrm{Tb}_{1-\mathrm{x}} \mathrm{Y}_{\mathrm{x}}\right)_{0.8} \mathrm{Sm}_{0.2} \mathrm{Fe}_{2}$ was done on highpure REM and $\mathrm{Fe}$ in arc furnace with non-expendable tungsten electrode on specially constructed copper water-cooled bottom plate in specially purified inert atmosphere (argon) at normal pressure. Then samples have undergone homogenizing annealing for 2 weeks.

$\mathrm{X}$-ray diffraction analysis showed that only quasi-binary alloys $\mathrm{Tb}_{0.8} \mathrm{Sm}_{0.2} \mathrm{Fe}_{2}, \mathrm{Y}_{0.8} \mathrm{Sm}_{0.2} \mathrm{Fe}_{2}$ are mono-phase and have structure of Laves cubic phase S15. More complex compounds Tb0.64Y0.16Sm0.2Fe2, $\mathrm{Tb}_{0.48} \mathrm{Y}_{0.32} \mathrm{Sm}_{0.2} \mathrm{Fe}_{2}, \mathrm{~Tb}_{0.32} \mathrm{Y}_{0.48} \mathrm{Sm}_{0.2} \mathrm{Fe}$ 2, $\mathrm{Tb}_{0.16} \mathrm{Y}_{0.64} \mathrm{Sm}_{0.2} \mathrm{Fe}_{2}$ had phase segregation. These samples had the second phase $\mathrm{RFe}_{3}$, whose amount varied from 5 to 15 $\%$.

The main research methods were: - polycrystalline X-ray diffractometry; - alloys magnetization measuring in the fields up to $15 \mathrm{Ke}$ in a wide temperature range with the help of pendulum magnetometer of Dominikali type;

- Magnetization measuring in strong magnetic fields with temperature interval $2-300 \mathrm{~K}$.

- Magnetostriction measuring with strain-gauge method.

\section{RESULTS}

Magnetic properties. It is known [8], that rare-earth Laves phases containing magnetic metals atoms can be both ferromagnetic as well as ferrimagnetic. More often they are considered as binary sub lattice magnetic substances, whose one sub lattice consists of rare-earth R-ions, and the second of 3d-transitional ions. Most $\mathrm{RM}_{2}$ compounds have collinear magnetic structures, whose magnetic moments of $\mathrm{R}$ and $3 \mathrm{~d}$ sub lattice are parallel to each other, with light rare-earth elements total magnetic moments $\mathbf{M}_{\mathrm{R}}$ and $\mathbf{M}_{3 \mathrm{~d}}$ of rare-earth and 3d-transitional sub lattice are similarly oriented, that is why absolute magnetic moment is: $\mathbf{M}_{\mathrm{RM} 2}=\mathbf{M}_{\mathrm{R}}+2 \mathbf{M}_{3 \mathrm{~d}}$.

For heavy rare-earth elements total magnetic moments are $\mathbf{M}_{\mathrm{R}}$ and $\mathbf{M}_{3 \mathrm{~d}}$ of rare-earth and 3d-transitional sub lattices are anti parallel, that is why absolute magnetic moment is: $\mathbf{M}_{\mathrm{RT} 2}=$ $\mathbf{M}_{\mathrm{R}}-2 \mathbf{M}_{3 \mathrm{~d}}$. 
In the first case, ferromagnetic magnetic ordering temperature is Curie temperature $T_{C}$, in the second case is Neel temperature $T_{N}$. As it is known, these are temperatures of magnetic phase transition "order-disorder" and their value is important for problem solution. Usually, Curie temperatures are determined by magnetization measurements. Alloys magnetization study $\left(\mathrm{Tb}_{1-\mathrm{x}} \mathrm{Y}_{\mathrm{x}}\right)_{0.8} \mathrm{Sm}_{0.2} \mathrm{Fe}_{2}$ was done in fixed magnetic fields $2 \mathrm{Ke}$ in temperature range from 77 to $300 \mathrm{~K}$. This temperature range is far from magnetic transition temperature - Curie temperature. Accourding to the reference books, Curie temperature of the compound $\mathrm{TbFe}_{2} \mathrm{Tc}=711 \mathrm{~K}$, for $\mathrm{SmFe}_{2}-\mathrm{Tc}=700 \mathrm{~K}$, and compound $\mathrm{YFe}_{2}$ has $\mathrm{Tc}=550 \mathrm{~K}$ [9]. That is why magnetization with small values was almost independent of temperature. Magnetization concentration dependence behavior at different temperatures is more interesting (pic. 1). It is clear, that in the range of studied temperatures the curves $\sigma(\mathrm{T})$ are close to each other.

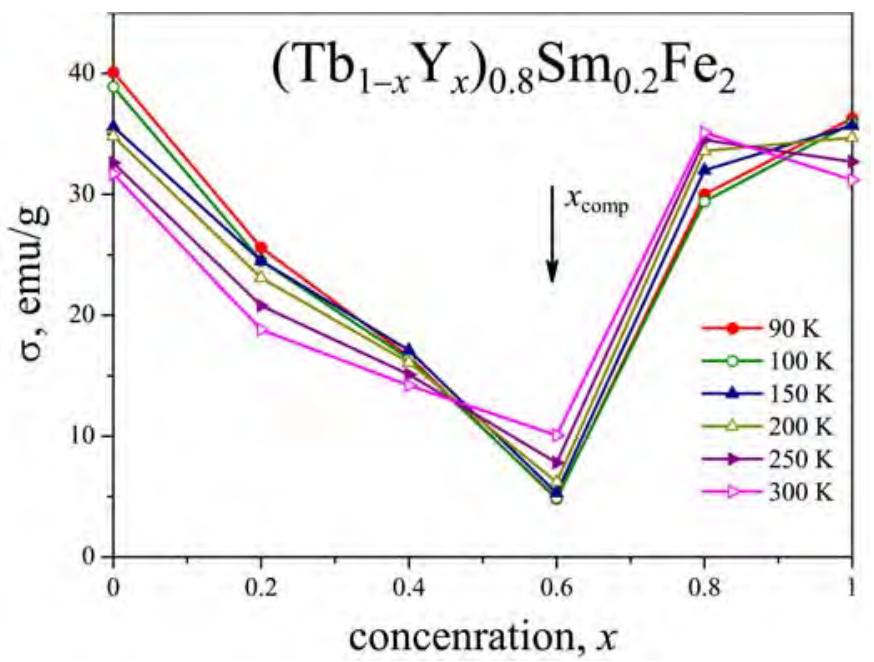

Fig. 1. Alloys magnetization dependence (Tb1-xYx)0.8Sm0.2Fe 2 on substitution parameter $\mathrm{x}$ in temperature range from 90 to $300 \mathrm{~K}$.

Herewith, in the range of parameter values $x=0.6$ in this alloys system there occurs magnetic compensation of rareearth and iron sub-lattices. In the area of this concentration magnetization almost equals zero. Further yttrium concentration upto $\mathrm{x}=0.8$ results in sharp magnetization increase up to the values almost equal to magnetization values of the initial compound $\mathrm{TbFe}_{2}$. Further yttrium content increase does not change magnetization in this field significantly.

Main magnetic characteristics of system alloys calculated from magnetization measurement results at $2 \mathrm{~K}$ are in table 1.
TABLE I. MAIN MAGNETIC CHARACTERISTICS OF THE ALLOYS $\left(\mathrm{TB}_{1-}\right.$ $\left.{ }_{\mathrm{X}} \mathrm{Y}_{\mathrm{X}}\right)_{0.8} \mathrm{SM}_{0.2} \mathrm{FE}_{2}$

\begin{tabular}{|c|c|c|c|c|c|}
\hline $\mathbf{x}$ & Alloys/Properties & $\begin{array}{c}\mathbf{6 s ,} \\
\mathbf{e m u / q}\end{array}$ & $\begin{array}{c}\mathbf{M}, \\
\mathbf{M B}\end{array}$ & $\begin{array}{c}\mathbf{M} \text { (Fe, } \\
\mathbf{M B}\end{array}$ & $\mathbf{T}_{\mathbf{c}}, \mathbf{K}$ \\
\hline 0 & $\mathrm{~Tb}_{0.8} \mathrm{Sm}_{0.2} \mathrm{Fe}_{2}$ & 74.05 & 3.56 & 1.75 & 672.1 \\
\hline 0.2 & $\mathrm{~Tb}_{0.64} \mathrm{Y}_{0.16} \mathrm{Sm}_{0.2} \mathrm{Fe}_{2}$ & 47.81 & 2.20 & 1.71 & 613.1 \\
\hline 0.4 & $\mathrm{~Tb}_{0.48} \mathrm{Y}_{0.32} \mathrm{Sm}_{0.2} \mathrm{Fe}_{2}$ & 33.42 & 1.47 & 1.355 & 601.7 \\
\hline 0.6 & $\mathrm{~Tb}_{0.32} \mathrm{Y}_{0.48} \mathrm{Sm}_{0.2} \mathrm{Fe}_{2}$ & 11.32 & 0.47 & 1.14 & 576.8 \\
\hline 0.8 & $\mathrm{~Tb}_{0.16} \mathrm{Y}_{0.64} \mathrm{Sm}_{0.2} \mathrm{Fe}_{2}$ & 50.30 & 2.02 & 1.66 & 568.3 \\
\hline 1 & $\mathrm{Y}_{0.8} \mathrm{Sm}_{0.2} \mathrm{Fe}_{2}$ & 64.13 & 2.45 & 1.16 & 539.5 \\
\hline
\end{tabular}

It should be noted that Curie temperatures values of the alloys in the given system are determined from the study results of the inverse susceptibility (pic. 2). Correspondence to the above mentioned values of Tc for initial compounds $\mathrm{TbFe}_{2}, \mathrm{SmFe}_{2}$ and $\mathrm{YFe}_{2}$ is rather good.

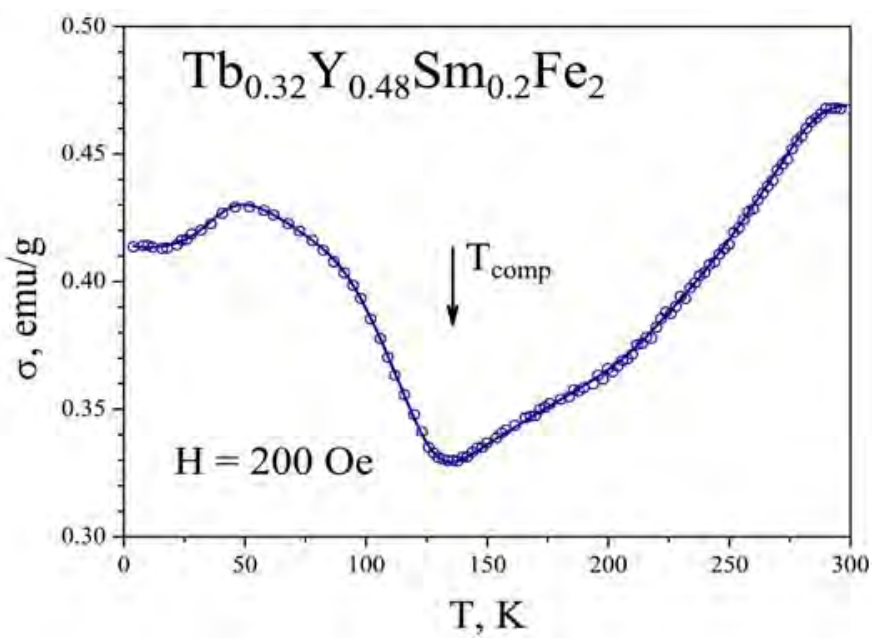

Fig. 2. Temperature dependence system alloys susceptibility $\left(\mathrm{Tb}_{1}\right.$. $\left.{ }_{\mathrm{x}} \mathrm{Y}_{\mathrm{x}}\right)_{0.8} \mathrm{Sm}_{0.2} \mathrm{Fe}_{2}$.

In order to acquire magnetization saturation and determine its real value, we studied alloys magnetization $\left(\mathrm{Tb}_{1-}\right.$ $\left.{ }_{\mathrm{x}} \mathrm{Y}_{\mathrm{x}}\right)_{0.8} \mathrm{Sm}_{0.2} \mathrm{Fe}_{2}$ in temperature ranges $2-200 \mathrm{~K}$ in magnetic fields $140 \mathrm{kE}(14 \mathrm{~T})$. For compounds with greater $\mathrm{Tb}$ magnetization saturation on curves $\sigma(\mathrm{H})$ is achieved in magnetic fields areas of $20 \mathrm{kE}$ order and magnetization saturation decreases slightly with temperature increase in temperature ranges up to $300 \mathrm{~K}$ (see pic. 3 ). 


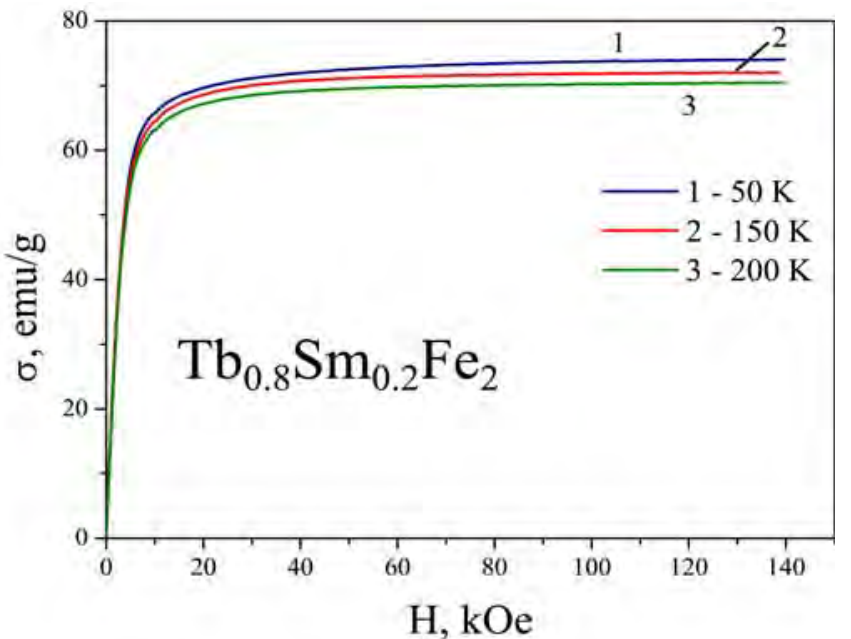

Fig. 3. Alloy magnetization dependence $\mathrm{Tb} 0.8 \mathrm{Sm} 0.2 \mathrm{Fe} 2$ on magnetic field intensity.

In compensating compound area with substitution parameter $x_{\text {comp }}=0.6$ there appears some variation in magnetization curves positioning. Here magnetization minimum value is for compensation temperature $\mathrm{T}_{\text {comp }}=136$ $\mathrm{K}$ for this compound (pic. 4).

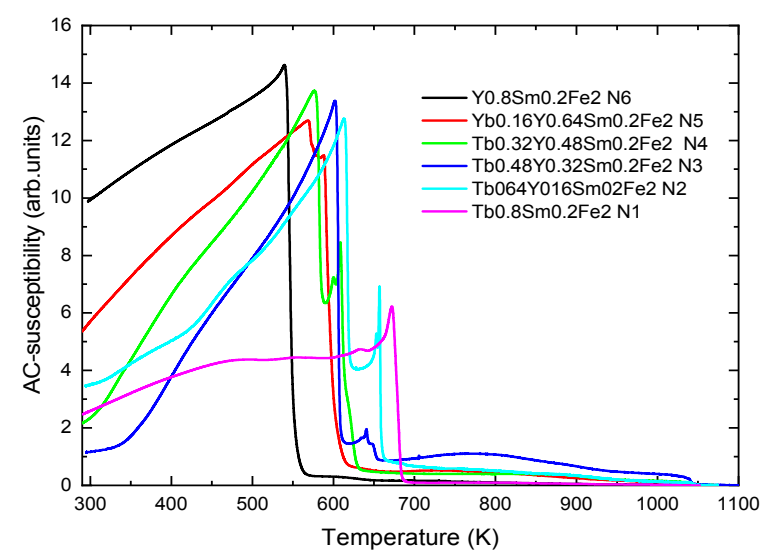

Fig. 4. Temperature dependence of alloy specific magnetization with substitution parameter $\mathrm{x}=0.6$ (alloy $\mathrm{Tb} 0.32 \mathrm{Y} 0.48 \mathrm{Sm} 0.2 \mathrm{Fe} 2$ ) in the field $\mathrm{H}=$ $200 \mathrm{E}$.

In substitution alloys during relative concentration changes in light ( $\mathrm{Y}$ and $\mathrm{Sm}$ ) and heavy $(\mathrm{Tb})$ rare-earth ions there is a conflict of exchange magnetic interactions "Tb-Fe" and "Sm$\mathrm{Fe}$ ", resulting in certain values of yttrium concentration $\mathrm{x}_{\text {Comp }}$ to their total mutual compensation and consequent change of magnetic order type: from ferrimagnetic to ferromagnetic. Thus substitution parameter value $x_{\text {comp }}=0.6$ is a point of magnetic phase transition ferrimagnitism -ferromagnetism: at $\mathrm{x}<\mathrm{x}_{\text {Comp }}$ - ferrimagnetic order of magnetic moments ordering of rare-earth and iron sub lattices at $\mathrm{x}>\mathrm{x}_{\mathrm{Comp}}$ is ferromagnetic.

Magnetostriction. Magnetostriction is a sensitive parameter to sign and value of exchange interactions.

Magnetostriction is change in body form and measurements during its magnetization. Magnetostriction is a dimensionless quantity. It equals relative change of magnetic dimensions with activated magnetic field $\mathbf{H}$. There exist Joule effect $\lambda_{\text {II }}$, when field intensity $\mathbf{H}$ coincides with measurement direction and cross magnetostriction, when the directions are mutually perpendicular $\lambda_{\perp}$.

Alloys magnetostriction system measurements were conducted in temperature ranges $80-300 \mathrm{~K}$ in magnetic fields up to $12 \mathrm{kE}$ with strain-gauge method. Picture 5 shows values of Joule effect and cross magnetostriction of system alloys depending on substitution parameters $\mathrm{x}$.

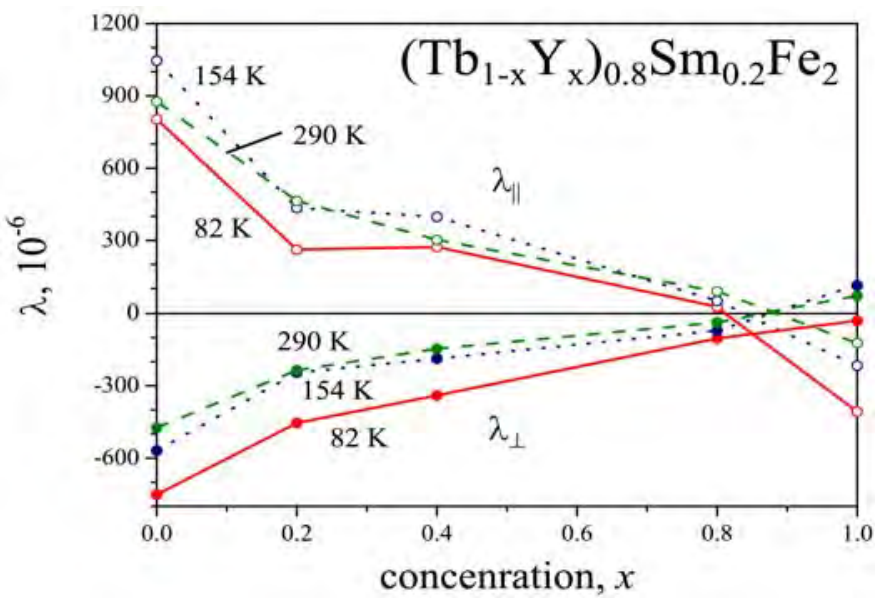

Fig. 5. Dependence of Joule effect and cross sectional magnetization of alloys $\left(\mathrm{Tb}_{1}-\mathrm{xYx}\right)_{0.8} \mathrm{Sm}_{0.2} \mathrm{Fe}_{2}$ on substitution parameter $\mathrm{x}$.

It is seen that Joule effect of all system alloys $\left(\mathrm{Tb}_{1-}\right.$ $\left.{ }_{\mathrm{x}} \mathrm{Y}_{\mathrm{x}}\right)_{0.8} \mathrm{Sm}_{0.2} \mathrm{Fe}_{2}$ with substitution parameters $\mathrm{x} \leq 0.8$ in outer magnetic field $\mathrm{H}=12 \mathrm{kE}$ is positive, and cross magnetostriction is negative. In alloys with value $x \geq 0.9$, on the contrary, Joule effect is negative, and cross magnetization is positive. That is in parameters values range $0.8 \leq \mathrm{x} \leq 0.9$ there is sign inversion of magnetostriction constants.

It should be noted, that sign inversion of magnetostriction constants was observed earlier for some rare-earth intermetallides [10].

\section{CONCLUSION}

The peculiarity of these compounds is that the compound $\mathrm{TbFe}_{2}$ demonstrates positive spontaneous and induced magnetostriction, while compound $\mathrm{SmFe}_{2}$ has negative magnetostriction. There is sign inversion of magnetostriction constants in system $(\mathrm{Tb}, \mathrm{Y}, \mathrm{Sm}) \mathrm{Fe}_{2}$ in the range of parameter values of substituting of terbium with yttrium $0.8 \leq \mathrm{x} \leq 0.9$.

Diluting rare-earth sub lattice with non magnetic yttrium and distance increase between terbium and samarium magneto active atoms results in sign change of conflicting exchange interactions $\mathrm{Tb}-\mathrm{Fe}$ and $\mathrm{Sm}-\mathrm{Fe}$ with separate yttrium concentration.

Moreover, varying concentration of rare-earth component (terbium) we can receive a compound with complete magnetic compensation of magnetization in all temperature range of magnetic ordering. 
And combination of light and heavy elements ions in one and the same rare-earth sub lattice (terbium and samarium) allowed determining compositions of such multi component system alloys ( $\mathrm{Tb}, \mathrm{Y}, \mathrm{Sm}) \mathrm{Fe}_{2}$, whose magneto crystalline anisotropy is almost compensated, consequently, we can expect a compound with more optimal magnetostriction characteristics [8]. It is an alloy with substitution parameter $x_{\text {comp }}=0.6$, that corresponds to yttrium concentration 48 at. $\%$. It corresponds to the following composition$\mathrm{Tb}_{0.32} \mathrm{Y}_{0.48} \mathrm{Sm}_{0.2} \mathrm{Fe}_{2}$. Composition of two factors mentioned above was not studied.

Compounds with these peculiarities of magnetic and magnetostriction properties can be used in such spheres as robotics technology.

\section{References}

[1] T.F. Grigorieva, A.P. Barinova, N.Z. Lyakhov, "Mechano - chemical synthesys of intermetallic compounds", Uspekhi khimii, Vol. 70, № 1, pp. 52-71, 2001.

[2] A.S. Ilushin, Structural physics fundamentals of rare-earth innermetallic compositions, Moscow, MSU, 2005.

[3] G. Engdahl, «Handbook of giant magnetostrictive materials», Academic Press, pp. $386,2000$.

[4] Jr.K.A. Gschneidner, V.K. Pecharsky, A.O. Tsokol, «Recent Developments in Magnetocaloric Materials», Rep. Progr. Phys, vol. 68, pp. 1479-1539, 2005.

[5] F. Stein, M. Palm, G. Sauthoff, «Structure and stability of Laves phases», Part I. Critical assessment of factors controlling Laves phase stability. Intermetallics. vol. 12, pp. 713-720, 2004

[6] L.A. Stashkova, V.S. Gaviko, N.M. Mushnikov, P.B Terentiev, «Hydrogen ordering in rare-earth intermetallic compositions (ErTd)Fe2 with giant spontaneous magnetostriction». Metal physics and Metal Science, V. 114, № 12, P. 1068-1074, 2013.

[7] W.J. Rent, J.L. Yang, B.Li.D. Li, X.G. Zhao, Z.D. Zhang, «Magnetostriction and magnetic anisotropy of ( $\mathrm{Sm}, \mathrm{Ce}) \mathrm{Fe}_{2}$ compounds», Physica, vol. B, 404, pp. 3410-3412, 2009.

[8] A.S. Ilushin, Ye.V. Solodov, Z.S Umkhaeva, «Structural and magnetic transformations in pseudobinary systems alloys ( $\mathrm{Sm} 1-\mathrm{xTbx}) \mathrm{Fe}_{2}$ », Perspektivnye Materialy, № 11, p. 42 - 47, 2013.

[9] H. Samata, T. R. Kasai, T. Taniguchi and Y. Nagata, «Al substitution effects to the magnetic properties of the compounds in the $\mathrm{Sm}-\mathrm{Fe}$ system», J. of Solid State Chemistry, vol. 171, pp. 339-344, 2003.

[10] [10] K. Tureka, J. Ruszb, M. Divis, «Electronic structure and volume magnitostriction of rare-earth metals and compounds», Journal of Magnetism and Magnetic Materials, pp. 357 - 363, 2005 